\title{
Isospora belli associated recurrent diarrhea in a child with AIDS
}

\author{
M. Nateghi Rostami • B. Nikmanesh • \\ M. T. Haghi-Ashtiani • M. Monajemzadeh • \\ M. Douraghi $\cdot$ Z. Ghalavand $\cdot$ L. Kashi
}

Received: 14 January 2013/ Accepted: 23 February 2013/Published online: 7 March 2013

(C) Indian Society for Parasitology 2013

\begin{abstract}
Persistent diarrhea is a major manifestation of Acquired Immunodeficiency Syndrome (AIDS) which might be more complicated in Human Immunodeficiency Virus (HIV) infected children especially those from developing countries. There are numerous reports showing the emergence of intestinal opportunistic coccidian parasites, mostly Cryptosporidium parvum and Isospora belli in HIV-infected individuals. The prevalence of isosporiasis is probably underestimated in developing countries because routinely not all HIV-infected patients are examined for the presence of this protozoan infection. Here we report a case of HIV-infected isosporiasis presenting with failure to thrive and persistent diarrhea. Since $I$. belli infection in children responds well to therapy with trimethoprimsulfamethoxazole, isosporiasis should be considered as a treatable infection in AIDS, if it is detected at proper time.
\end{abstract}

\section{Nateghi Rostami}

Department of Public Health, Faculty of Health, Qom University

of Medical Sciences, Qom, Iran

e-mail: rostami52@yahoo.com

B. Nikmanesh $(\varangle) \cdot$ M. T. Haghi-Ashtiani .

M. Monajemzadeh $\cdot$ L. Kashi

Department of Pathology, Children's Medical Center, Tehran

University of Medical Sciences, No. 62, Dr. Gharib St.,

Keshavarz Blvd., Tehran, Iran

e-mail: bahram_nikmanesh@yahoo.com

M. Douraghi

Division of Bacteriology, Department of Pathobiology,

School of Public Health, Tehran University of Medical

Sciences, Tehran, Iran

Z. Ghalavand

Department of Microbiology, Shaheed Beheshti University

of Medical Sciences, Tehran, Iran
Keywords Isospora belli - Failure to thrive · AIDS . Diarrhea

\section{Introduction}

Opportunistic infections account for cases of severe and persistent diarrhea in immunocompromised individuals. Isospora belli, one of the human coccidian parasites, is among the most commonly identified causes of chronic diarrhea in patients with Acquired Immunodeficiency Syndrome (AIDS). Isosporiasis is primarily considered a waterborne and/or a foodborne disease. I. belli infection in immuocompetent hosts is often associated with a selflimited watery diarrhea accompanied with abdominal pain, steatorrhea, and peripheral eosinophilia. In children, especially those with AIDS or other immunodeficiencies, clinical outcome may be more severe, characterized by dehydration, malabsorption, weight loss, nausea, vomiting, fever and malaise (Arora and Arora 2009; Mirdha et al. 2002). Since coccidian parasites are important opportunistic infections in AIDS, it is crucial to have as much information as possible regarding the manifestations and management of these infections especially in children. Here a case of HIV-infected isosporiasis presenting with failure to thrive (FTT) is reported.

\section{Case report}

In February 2009, a 3 year-old girl was admitted with FTT with a $4 \mathrm{~kg}$ weight loss during 2-3 months, low-grade fever, and severe diarrhea. She experienced episodes of recurrent diarrhea from 5 months ago with 1 week relief intervals. Physical examination was normal except for white patches 
on the mouth palate. Biopsy of esophageal and duodenum showed moderate esophagitis and dilated lymphatic channels in lamina propria of duodenum suggestive of lymphangiectasis. Cystic fibrosis and celiac disease were ruled out in the patient by laboratory methods. Leukocyte count, $\mathrm{Hb}$ and platelet count were in normal range. Erythrocyte sedimentation rate (ESR) was varied between 37 and $45 \mathrm{~mm} / \mathrm{hr}$. Liver function tests (LFT) were slightly increased, with ALT of $68 \mathrm{mg} / \mathrm{dl}$ (normal up to 40) and AST of $97 \mathrm{mg} / \mathrm{dl}$ (normal up to 40), but ALP was $318 \mathrm{mg} / \mathrm{dl}$ which existed in normal range $(180-1200 \mathrm{mg} / \mathrm{dl})$. Routine stool examination for ova and parasites and stool culture for pathogenic coliforms were negative. Sudan black staining of stool smear showed significant fat droplets and moderate number of yeast bodies. Treatment was started with cefexim, gentamycin, metronidazole and fluconazole, and then followed by amikacin and penicillin. After 2 weeks, the patient ameliorated and discharged with orally administered trimethoprim-sulfamethoxazole (TMP-SMX) and medium chain triglycerides (mct oil) regimen.

After 13 months, the patient again was admitted with malabsorption and abdominal discomfort. She suffered from fever, productive cough, wheezing, violent mucoid diarrhea, steatorrhea, repeated foul smelling feces and emaciation. Endoscopy showed esophageal ulceration, exodative otitis was noticed at physical examination, and chest X-ray showed paratracheal bilateral consolidation. Human Immunodeficiency Virus (HIV) Ab was positive using ELISA method which was confirmed by western blotting technique. $\mathrm{HBs} \mathrm{Ag}$ and $\mathrm{Ab}, \mathrm{HBc} \mathrm{Ab}$, and $\mathrm{HCV} \mathrm{Ab}$ all were negative. $\mathrm{CD} 4 / \mathrm{CD} 8 \mathrm{~T}$ cell ratio was reduced to as much as $0.2 \%$ down to $0.09 \%$. The relative count of CD4 + cells ranged from 6.9 to $9 \%$ of total lymphocytes. Also, CD19 and CD20 positive cells were reduced to 1.8 and $1.9 \%$ of total lymphocyte, respectively. Laboratory examinations showed that leukocyte count was $5.3 \times 10^{9} / 1$ and platelet count dropped to $38 \times 10^{9} / 1$. Also, laboratory findings showed hyper $\gamma$-globulinemia and increased liver enzymes as AST $127 \mathrm{mg} / \mathrm{dl}$, ALT $59 \mathrm{mg} / \mathrm{dl}, \gamma$-GT $184 \mathrm{IU} / \mathrm{l}$ (normal range: 7-60) and bilirubin in normal range. There was history of sibling death as a result of pulmonary infection and dysentery at 8 month old age. The patient's mother, a 55 years old white woman was infected with HIV through heterosexual transmission from her husband who was working as a truck driver and had been diagnosed with AIDS. The stool specimen was macroscopically watery and permanent stained smear with modified ZiehlNeelsen acid fast method showed many I. belli oocysts, measuring on average $27 \times 13 \mu \mathrm{m}$ in diameter (Fig. 1) Careful search failed to reveal other coccidian parasites such as Cryptosporidium spp. infection. Small bowel follow-through showed severe hypertrophy of duodenal and jejunal mucosa and normal ileum.

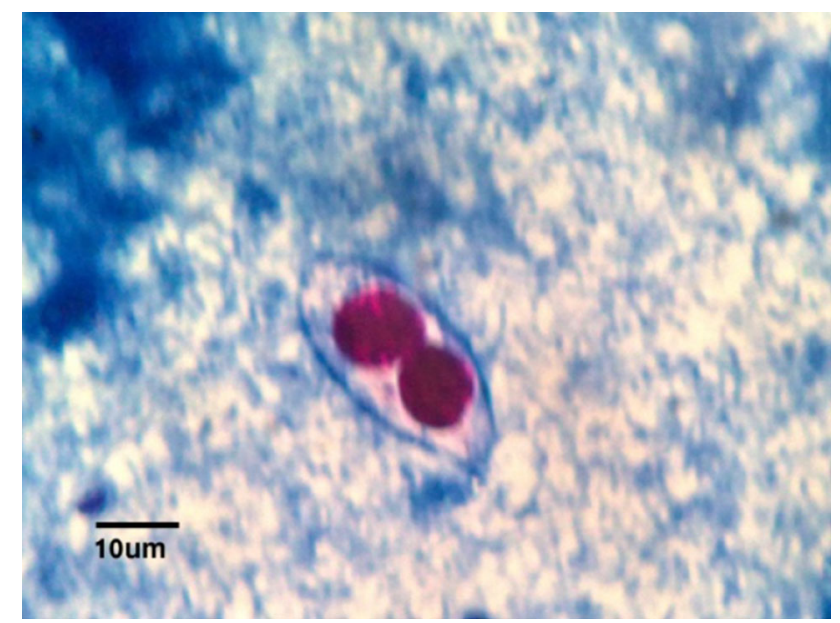

Fig. 1 Isospora belli oocysts on stool smear stained using ZiehlNeelsen method

She was put under the treatment of vancomycin, cefotaxime, ceftazidim, and TMP-SMX. The acid-fast staining of stool specimens turned negative for I. belli oocyst after TMP-SMX treatment completion. She discharged after 9 days by her parents request and consent, and unfortunately died after 1 month.

\section{Discussion}

Isospora is worldwide distributed particularly in the tropical and subtropical regions (Arora and Arora 2009; Gupta et al. 2008). Very limited information is available on isosporiasis infection from Iran, few cases were reported in a recent study of 356 HIV-positive patients (Agholi et al. 2013) and as a case report (Hazrati Tappeh et al. 2001). Different studies have shown I. belli is the most common parasite in HIV-positive patients which was strongly associated with diarrhea. In a study of 245 diarrheal stool specimens from HIV-infected individuals from India, who received prophylactic treatment with TMP-SMX, parasitic infections were observed in $37 \%$ of cases, while I. belli $(26.1 \%)$ was the most common parasite followed by Entamoeba spp. (3.3\%), Cryptosporidium parvum (2.9\%), and Giardia lamblia (1.6\%) (Vignesh et al. 2007). The same results were found by others who reported $I$. belli in $17 \%$ (16/94) (Joshi et al. 2002) and in $41.1 \%$ (14/34) HIV seropositive patients with chronic diarrhea (Gupta et al. 2008). In the latter study I. belli was also seen in $6.3 \%$ (5/ 79) of non-diarrheal cases. In another study of HIV-positive patients $(n=397)$ diarrhea was reported in $98 \%$ of the cases, of whom $14 \%(n=56)$ was infected with I. belli (Certad et al. 2003). The presence of persistent diarrhea is a common feature of AIDS. In Zaire, results of a cohort study of 429 infants born to mothers with HIV+ 
showed HIV + infants comparing HIV - infants had greater risk of development of recurrent and/or persistent diarrhea and 11-fold increased risk of mortality due to diarrhea (Thea et al. 1993). In our patient, abdominal discomfort was concomitant with steatorrhea which is a common complication of isosporiasis, as in the report of Brandborg e al. (1970) of six $I$. belli infections that all presented with steatorrhea. Our patient was admitted with malabsorption and weight loss and eosinophil count not exceeded $4 \%$ on average. Study of HIV-infected patients showed that eosinophilia was strongly associated with isosporiasis; however higher eosinophil counts were described in I. belli infected patients without weight loss (Certad et al. 2003). This finding could be described that in patients with considerable weight loss the HIV disease is more severe and defect of the immune system may involve the production of eosinophils (Arora and Arora 2009). Celiac was not confirmed in our patient, however celiac disease is one of the most frequent causes of malabsorption as reported previously (Behera et al. 2008). Isosporiasis might manifest itself as a systemic disseminated infection in patients with AIDS, I. belli was detected within the cells of mediastinal, periaortic and mesenteric lymph nodes at autopsy (Restrepo et al. 1987), and in gallbladder biopsy samples of a patient presenting with cholecystitis (Benator et al. 1994). A number of studies have reported $I$. belli as an etiologic agent of diarrhea in other groups of immunodeficient patients (Koru et al. 2007; Meamar et al. 2009; Resiere et al. 2003).

Because chronic diarrhea in patients infected with HIV results in a significant morbidity and mortality, the reliable diagnosis and management of diarrhea associated opportunistic infections in patients with AIDS is very important in preventing sever complications. Isosporiasis should be regarded in children presenting with FTT and in HIVinfected children with chronic watery diarrhea and weight loss.

Conflict of interest The authors declare that they have no conflicts of interest.

\section{References}

Agholi M, Hatam GR, Motazedian MH (2013) HIV/AIDS-associated opportunistic protozoal diarrhea. AIDS Res Hum Retrovir 29:35-41

Arora DR, Arora B (2009) AIDS-associated parasitic diarrhea. Indian J Med Microbiol 27:185-190

Behera B, Mirdha BR, Makharia GK et al (2008) Parasites in patients with malabsorption syndrome: a clinical study in children and adults. Dig Dis Sci 53:672-699

Benator DA, French AL, Beaudet LM, Levy CS, Orenstein JM (1994) Isospora belli infection associated with acalculous cholecystitis in a patient with AIDS. Ann Intern Med 121:663-664

Brandborg LL, Goldberg SB, Breidenbach WC (1970) Human coccidiosis-a possible cause of malabsorption. $\mathrm{N}$ Engl $\mathrm{J}$ Med 283:1306-1313

Certad G, Arenas-Pinto A, Pocaterra L et al (2003) Isosporiasis in Venezuelan adults infected with human immunodeficiency virus: clinical characterization. Am J Trop Med Hyg 69:217-222

Gupta S, Narang S, Nunavath V, Singh S (2008) Chronic diarrhea in HIV patients: prevalence of coccidian parasites. Indian J Med Microbiol 26:172-175

Hazrati Tappeh Kh, Mohammad Zadeh H, Mohammedi A, Yousefi MH (2001) A case report of Isospora belli from west Azerbayjan province, Iran. Urmia Med J 12(3) (Persian)

Joshi M, Chowdhary AS, Dalal PJ, Maniar JK (2002) Parasitic diarrhea in patients with AIDS. Natl Med J India 15:72-74

Koru O, Araz RE, Yilmaz YA et al (2007) Case report: Isospora belli infection in a renal transplant recipient. Turkiye Parazitol Derg 31:98-100

Meamar AR, Rezaian M, Mirzaei AZ et al (2009) Severe diarrhea due to Isospora belli in a patient with thymoma. J Microbiol Immunol Infect 42:526-529

Mirdha BR, Kabra SK, Samantray JC (2002) Isosporiasis in children. Indian Pediatr 39:941-944

Resiere D, Vantelon JM, Bourée P et al (2003) Isospora belli infection in a patient with non-Hodgkin's lymphoma. Clin Microbiol Infect 9:1065-1067

Restrepo C, Macher AM, Radany EH (1987) Disseminated extraintestinal isosporiasis in a patient with acquired immune deficiency syndrome. Am J Clin Pathol 87:536-542

Thea DM, St Louis ME, Atido U et al (1993) A prospective study of diarrhea and HIV-1 infection among 429 Zairian infants. N Engl J Med 329:1696-1702

Vignesh R, Balakrishnan P, Shankar EM et al (2007) Short report: high proportion of isosporiasis among HIV-infected patients with diarrhea in southern India. Am J Trop Med Hyg 77:823-824 